\title{
Developing MLS Location-Based Service Pilot System
}

Jouni Markkula, Artem Katasonov, and Artem Garmash

Information Technology Research Institute, University of Jyväskylä

P.O. Box 35, FIN-40351 Jyväskylä, Finland

E-mail:markkula@jyu.fi,artem.katasonov@titu.jyu.fi,artyom.garmash@titu.jyu.fi

\begin{abstract}
Development of mobile networks and terminals provides basis for advanced mobile services and applications. One of the new features is support for locating the mobile user by her/his terminal. The Location-Based Services (LBS), utilising this feature, are expected to form an important class of the new services. At the Information Technology Research Institute, University of Jyväskylä, LBSs have been studied in MultiMeetMobile research project. The project developed an LBS pilot system, called MLS. This paper presents the experiences collected during the development of the LBS pilot and describes the MLS system.
\end{abstract}

Keywords: Location-based services, mobile computing, XML, Java

\section{INTRODUCTION}

In the past few years, the mobile networks and terminals have been under intensive development. Voice capabilities have been augmented with data capabilities of increasing speed and other new features have been combined to the networks and terminals. One of these features is location support. The mobile user can be located by her/his mobile terminal, and the location can be transmitted to third parties offering mobile Location-Based Services (LBS). According the forecasts, the LBSs will have over 300 million users by the end of the year 2004 (Singhal et al. 2001).

At the Information Technology Research Institute, University of Jyväskylä, the research on LBS was started in summer 1999 in MultiMeetMobile project. The MultiMeetMobile project produced also a

The original version of this chapter was revised: The copyright line was incorrect. This has been corrected. The Erratum to this chapter is available at DOI: 10.1007/978-0-387-35584-9_19 
pilot system called MultiMeetMobile Location-based service System (MLS). MLS is a research prototype for studying LBS in emerging location-aware mobile environment.

The development of MLS was started in autumn 1999. At that time, the mobile data services for general public were practically non-existent. The WAP environment development was in its first stage; no WAP terminals were publicly available. Location Services (LCS) and positioning methods were specified for public mobile networks (GSM), but they were not yet implemented by any operator. After a while, the first map services on WAP were introduced. However, they were not very useful, because of the limitations of WAP environment. It took about two years, until summer 2001, before the first LBSs using network based positioning (cell area) were publicly available and the first "navigation phone", Benefon Esc! (Benefon), using GPS positioning, was on the markets.

LBSs are still in the early stage. The services in Europe are rather simple and mostly relying on text based (SMS or WAP) output. The services and applications utilising maps in mobile environment are using server centric approach and bitmap graphics for transmitting the geographic information to the mobile terminals. For example Benefon Esc! requires the user to download the (expectedly) needed bitmap maps beforehand to the phone through PC computer. The purpose of MLS was to concentrate on problems and features of more advanced and usable mobile LBSs that will be expectedly available within a few years time.

This purpose of this paper is to present some key features of LBS system development on the basis of the collected experiences in MultiMeetMobile project's research and development work. It presents the framework of the development, design principles and features of MLS, and describes the current stage of the system.

\section{FRAMEWORK OF MLS DEVELOPMENT}

MLS was aimed to be a general LBS system for supporting locationbased information access in wireless client-server environment. In the development, the properties and restrictions of distributed mobile computing environment were taken into account.

Mobile computing environment is formed of mobile network infrastructure and mobile terminals. The targeted network infrastructure is public land mobile network, supporting data transmission and locating of the mobile terminals. In Europe, it is current GSM data, HSCD, GPRS, and emerging EDGE and UMTS networks. The targeted terminals are current and evolving personal pocketsize mobile terminals, combined with mobile 
network access. The expectation is that the terminals will have increasing computing power and reasonable input/output features. Advanced LBSs with map display require new generation of communicators and smatphones with high-resolution ( $120 \times 120$ or more) colour displays.

Mobile computing environment has certain features that impose restrictions (see for example Garmash, 2000). The properties of mobile networks are: (relatively) low bandwidth, high bandwidth variability, long latency, unpredictable disconnections and communication autonomy. The properties of mobile terminals are: small and low-resolution displays, limited input capabilities, limited computing power, limited power, small memory size, vulnerability, varying design forms.

On the other hand, the advantage of mobile computing environment is that it allows the user to access and use information in any place at any time, especially en route, on the move. The LBSs and other mobile services are particularly useful for conducting suddenly emerging "here and now" tasks which are urgent, well specified, restricted and tied to the present location and context.

The practical conditions, when and where the mobile devices are used, bring also additional restrictions. These restrictions have to be taken very carefully into account when designing usable mobile services. The using conditions cannot be expected to be constant, as usually is the case in "desktop" conditions. The mobile users are typically in very unstable environment in varying conditions, where their cognitive capacity is demanded for other tasks too. For that reason, the applications and service access should be very simple and user friendly and the amount of presented information content limited and well specified.

The content of LBSs can be divided into two components: digital map data and location-based information. The distinction is essential, because the nature and application of these data types is different. Typically they also come from different sources, possessed by different actors in the service architecture.

Digital map data is the geographic base data that form the geographic data infrastructure of LBSs. It is used by most of the services. Digital map data consists of general geographic features that are used for locational reference and spatial analysis; such as road networks and administrative areas. This base data is relatively stable in time and it is often maintained by public sector, e.g., municipalities or national mapping agencies. For example in Finland the National Land Survey is responsible of official mapping and Finnish Road Administration is preparing national road and street network database, DIGIROAD (Liikenne ja viestintäministeriö, 2001) for new location-based and mobile services. The digital map data used in LBSs are based mostly on the existing GIS databases. 
In contrast to geographic base data, location-based information forms the value-added content of LBSs. Location-based information consists of any data that can be georeferenced, associated to location; for example location of the restaurants or weather forecast in a particular area. It is the content that various LBS providers are offering to mobile customers, often combined with the digital map data. Location-based information can be relatively static (e.g. the restaurants) or it can be rather dynamic (e.g. the weather). This data from different content providers can be either stored in GIS databases or dynamically created and geocoded by the service.

\section{DESIGN PRINCIPLES AND FEATURES OF MLS}

MLS was developed as a research prototype for general LBS system that is using maps for information presentation and access at the terminal. The approach was to take into account the properties and restrictions of mobile computing environment from the beginning of the development.

The starting point was a view of an advanced and usable LBS system supporting navigation and location-based information access operating on the target environment outlined in the previous section. The LBS system was considered to be utilising the two types of content, digital map data and location-based information. The information content can be combined from different heterogeneous data sources. In addition, the LBS system should support some analysis functions, such as spatial and non-spatial queries and route finding. In that sense a LBS can be considered as a specialised Geographic Information System, GIS for a mobile use (Burrough and McDonnell, 1998).

The MLS system was designed to be "GIS supported", utilising existing geographic databases and GIS methods. The relationship between LBS and GIS and possibilities of GIS to support development of advanced LBS systems were studied in MultiMeetMobile project (Virrantaus et al. 2001).

The digital map data for MLS was provided by the National Land Survey of Finland. In MLS the data is stored in Oracle database in geographic vector data format. MLS utilises also methods of geographical data analysis and presentation developed in GIS. The special features of MLS are presented below.

Geographic vector data. The MLS system transmits geographical data from the server to the client in a geographic vector data format. In this the MLS deviates from most of the existing LBSs, which relay on bitmap graphics. The vector geographic data allows modification of the data presentation. But, most significantly, it makes possible to move some computation from the server to the client. It allows, for example, utilisation 
of analysis functions on the client side, independently and without straining the server. Some of the advantages are the following:

The vector model explicitly describes geographic features. This allows linking spatial data and attributes. Therefore, a map can be accompanied with various information associated to objects.

In the vector model, the explicit description of the road network topology is possible. This allows implementation of route finding algorithms.

Vector data enables the use of advanced, real-time generalisation routines.

- Vector data enables the user to customise a map by defining colours and symbols as well as excluding some data themes.

V Vector data, in many cases, requires less storage space than data in the raster form.

Delegation of computation to the client. MLS design makes possible to delegate some of the computation within the system to the client. For example, various queries, such as route finding or searching of the nearest points of interest, could be done in the client. Processing power of the new mobile terminals usually allow processing of limited volumes of data in a reasonable time. Frequent queries from the client also cause extensive use of mobile network, and possibly a greater latency because of the limited bandwidth of the network.

Extensible Markup Language (XML). The MLS system uses XML encoding for geographic data. The MLS uses two types of XML-based geographic data formats: the standard Geography Markup Language (GML), specified by OpenGIS Consortium (OpenGIS, 2001); and Geographical Mobile Markup Language (GMML), developed in the MultiMeetMobile project (Garmash, 2000). There are some reasons for using self-developed GMML. Initially, the standard GML specifications were not publicly available when GMML was developed. The MLS also prefers more lightweight data format for transmitting and using within the client. In addition, GML has certain limitations; it does not support explicitly network topology information. Network topology is necessary for many typical navigation applications, such as route finding. Currently the most recent GML version 2.0 enables implicit modelling of topology by the XLink mechanism. Better tools for topology are also promised in the future.

Moreover, MLS system utilises XML as the core technology for data representation. XML-based data formats can be used in interaction with mobile devices and content servers. XML-based approach offers the following advantages: 
Standard tools, such as XML parsers and XSLT processors, can be used for extracting and transforming data.

XML allows data integration from heterogeneous sources. Data from different geographic data and information content providers can be integrated into one LBS system.

$\square \quad$ XML facilitates interoperability between LBS components.

Different data formats for different target platforms can be used.

Java. The client application of the MLS system is implemented in Java language. Support for Java technology is an important feature of modern mobile devices. Currently, all the Symbian EPOC and Microsoft Windows based pocketsize computers support Java. In Japan the new mobile phones are able to run Java programs. Java support is included also in a version of Symbian EPOC 6 operating system, Pearl, aimed for next generation smartphones. Currently, Java support is expected to be a standard feature of practically all of the future mobile terminals. Java language presents a powerful and flexible tool for mobile services development because of its platform-independence. Client-side software developed in Java can be run in nearly every device supporting this technology, thus eliminating the need for development of different versions for every target platform. The MLS client application is lightweight, and after rewriting the user interface, it could be run even under Java 2 Micro Edition (J2ME) used in smartphones.

Intelligent data selection. MLS system is capable of selecting geographic data that is relevant for a specific user at a specific time. It uses a user-centred approach. The location of the user and his preferences are taken into account. The MLS client allows the user to specify a set of values defining the preferred scale of map and the relative importance of data themes. The MLS server then uses this information together with data on the location (as well as the direction and the speed of movement, if available) of the user for calculating importance of every geographic feature. The importance of a feature then defines if it will be represented, and, if so, the level of detail LOD this feature will have (some attributes can be excluded and the geometric form simplified). The total data volume is kept constant. Progressive data transmission is also supported. This means that if for a geographic feature LOD2 < LOD1 then the data set representing this feature at LOD2 is a subset of the data set at LOD1. No new data is introduced during generalisation, what can occur if, for example, smoothing of the geometric form or generalisation of attributes values are used. Therefore, increasing the level of detail for a feature, we have only to transmit additional data, no retransmission of already sent data or deletion of any data is needed. The MLS server stores the current LOD value for every 
geographic feature thus keeping track of data that user has currently available in the memory of his mobile terminal.

The objectives of such an approach are the following:

$\square$ Increase of the service usability, while only relevant data is transmitted

to the user and unnecessary is filtered out.

- Reduction of the volume of transmitted data.

Details of the used computational algorithm can be found in Katasonov (2001).

Transaction management support. In order to protect itself from the unexpected disconnections and crashes, the MLS client application keeps track of the history of interaction with the server (what data was sent and received) and stores all the data needed for recovery. This saves the application from information losses and guarantees basic transactional properties.

\section{RELATED WORK}

Currently, the market provides a set of mobile mapping applications and services. Some of them are born in the GIS field and represent simplified GIS systems running on mobile devices, like ESRI's ArcPad. Some others represent rather navigational LBSs providing the user with maps and possibly other data, like Webraska system (Mitchell, 2001) accessible with a WWW or a WAP browser.

While existing systems rely primarily on bitmaps or some applicationspecific formats, XML is positioned currently to be a standard way for encoding geographical data in future, including data transmitted to LBS clients. OpenGIS Consortium developed GML language (OpenGIS, 2001) that is going to be probably a standard. LaserScan developed a GMLcompatible Spatial Object Transfer Format (SOTF) (Mims et al., 2001) supporting incremental updates of data and topology information. XMLrelated work is going on in Finnish Geodetic Institute. In Lehto (2000), the applicability of XML and related technologies for geospatial applications is argued: using XSLT for transforming geographical data, XML Schema for spatial data modeling, XLink for description of spatial relationships. In Lehto and Kilpelainen (2001), an approach and a prototype system for realtime generalization of XML-encoded geographical data using XSLT are presented. Location Interoperability Forum (LIF) is developing currently a standard interface to location services (LCS) that is also XML-based.

Active research is going on concerning various aspects of mobile GIS. TeleCartography project of the Department of Cartography in Vienna University of Technology studies LBSs and impact of new technologies like 
UMTS on them (Gartner et al., 2001). Research work in LaserScan is related to on-demand generalisation of geographical data before transmitting to a mobile device; approach is based on the active-objects technology (Hardy et al., 2001). Research in University of California in Santa Barbara considers questions related to human-computer interaction in mobile GIS, usability of their user interfaces and use of augment reality technology (Clarke, 2001). Reichenbacher (2001) presents a general view on mobile cartography, which emphasises a need for transmitting data in the vector form, adaptive map rendering depending on the context and preferences of the user.

\section{DESCRIPTION OF MLS AT THE CURRENT STAGE}

\subsection{MLS architecture}

MLS is a general LBS system for mobile users offering a map and navigation service accompanied with access to location-based information. Architecture of the MLS system (figure 1) follows the general LBS architecture and includes the following main components:

Mobile terminal. Used for accessing the service and running the MLS client application.

- Mobile network. Used for communication between mobile devices and the MLS server.

$\square$ Location service (LCS). Provides information on the location of the mobile users. The dotted arrows between the mobile device and the LCS in figure 1 indicate that the mobile terminal could interact with an LCS directly and then send retrieved data to the MLS server in parameters of a query.

MLS server. Runs the MLS server application.

- Content servers. Offer information or other content that is used by the service and possibly transmitted to mobile users.

In MLS, the two types of content, digital map data and locationbased information, are differentiated, as seen in figure 1 . In the MLS system, the two content data sets are used. The first data set consists of the street network and the second data set consists of points of interest information. As we stated in the previous section, XML-based interaction with content servers is to be used. However, it is not implemented on the current stage. The knowledge about the structure of the content databases, as well as 
about the DTD and the meaning of the tags of the output GMML format, is embedded into the server application code. The MLS server retrieves and handles data on the base of this knowledge. Oracle database (with Oracle Spatial) is used for storing the data.

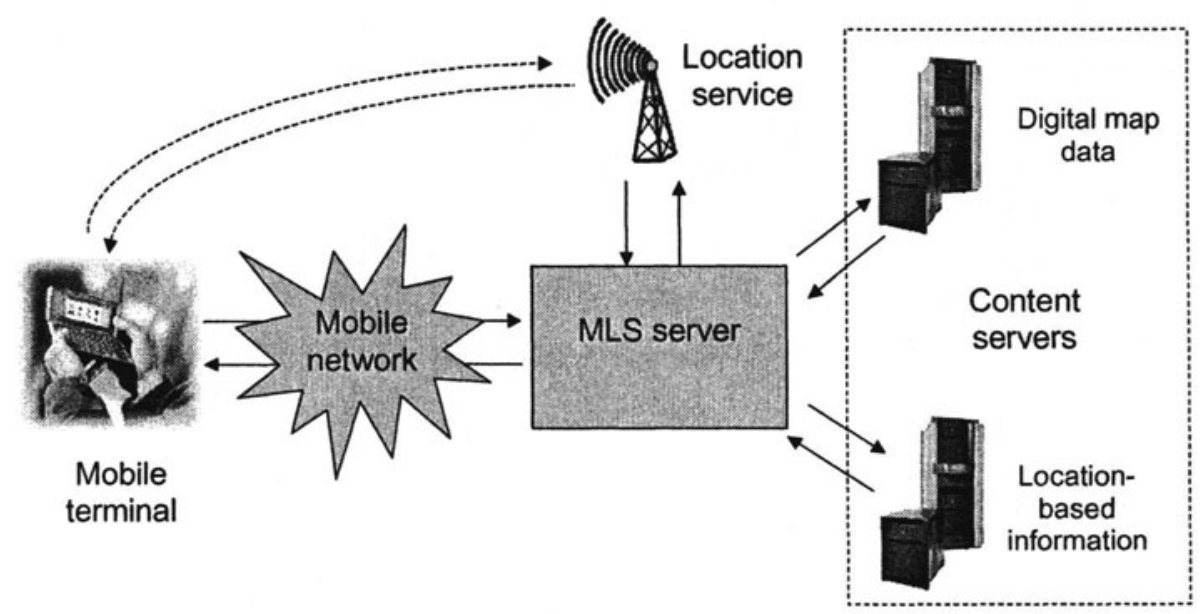

Figure 1. MLS Architecture

\subsection{The MLS server}

The server of the MLS system is an application accessible through a TCP/IP network. It is implemented in Java. Client-server interaction is implemented on the top of HTTP protocol. The MLS server supports authentication of users and three types of requests: request for a list of available data themes, request for data, and request for the location of the user (data is retransmitted from an LCS). Server response is encoded using GMML format. When handling a request for data, the location of the user and the parameters specified by him are taken into account for selection of relevant data. GMML data is transmitted in a compressed form using standard GZIP algorithm. This reduces the volume of the transmitted data about ten times. Use of algorithms developed specially for compression of XML data have also been studied.

The MLS server supports authentication of the users. For an authenticated user, a working session is opened. The following consecution of requests from the user is considered as belonging to this session. The session can be closed either by user's request or automatically, if there is no requests from the user for a certain time. 


\subsection{The MLS client}

The MLS client application is responsible for data representation and for the execution of the queries. The queries can be, for example, search for the nearest point of interest of a specified type. The client is implemented in Java. It can be run in nearly every device supporting Java technology. However, modifications in the user interface would still be necessary, for example, for porting it to Java 2 Micro Edition (J2ME) used in smartphones. For this reason, the architecture of the client application was designed to support further porting to other platforms.

The architecture of the MLS client uses Model/View/Controller (MVC) user interface paradigm (Krasner and Pope, 1988), in order to separate the logic of the application from the code controlling presentation.

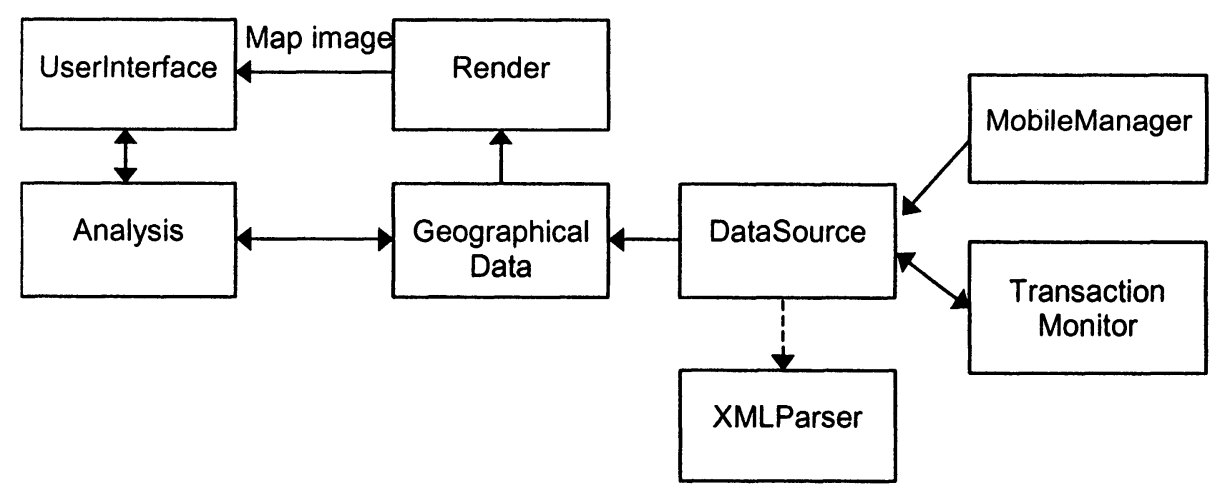

Figure 2. Architecture of the MLS client

Figure 2 depicts the main components of the MLS client application. The DataSource component interacts with the server in order to retrieve data and uses the XMLParser to process it. Based on obtained data, an internal model is constructed. This model is stored by the GeographicalData component. A Render component prepares a map image that is presented to the user. The map image is displayed by the UserInterface subsystem. The GeographicalData component represents the model. A Render and a UserInterface together form a representation. The UserInterface is responsible also for the user input and modification of the representation. Modification of the model is performed by the DataSource component. MobileManager component is responsible for updating the positions of mobile objects. However, the user is the only mobile object in MLS at the current stage. TransactionMonitor component is responsible for management of mobile transactions. Therefore, the model was maximally separated from 
platform-dependent components. For a new platform, the UserInterface component has to be mostly rewritten. In addition, some modifications probably have to be made to the DataSource component related to interaction with the server through a network (at least it is needed for porting to J2ME).

The MLS client application uses third-party libraries for handling XML. Microstar XML parser implementing SAX interface and kXML API are currently used. $\mathrm{kXML}$ is a lightweight XML API optimised for the Java 2 Micro Edition. kXML was originally developed at the University of Dortmund, now evolving further as a part of Enhydra project (kXML).

The following series of figures presents the appearance of the MLS client application on the Symbian EPOC system and its functionality at the current stage. The user starts with connecting. First, the location of the mobile device is requested from the LCS. Then a transaction with the MLS server begins. User is requested to authenticate himself. If authentication is passed, the client application requests from the MLS server a list of available data layers and presents it to the user (figure 3).

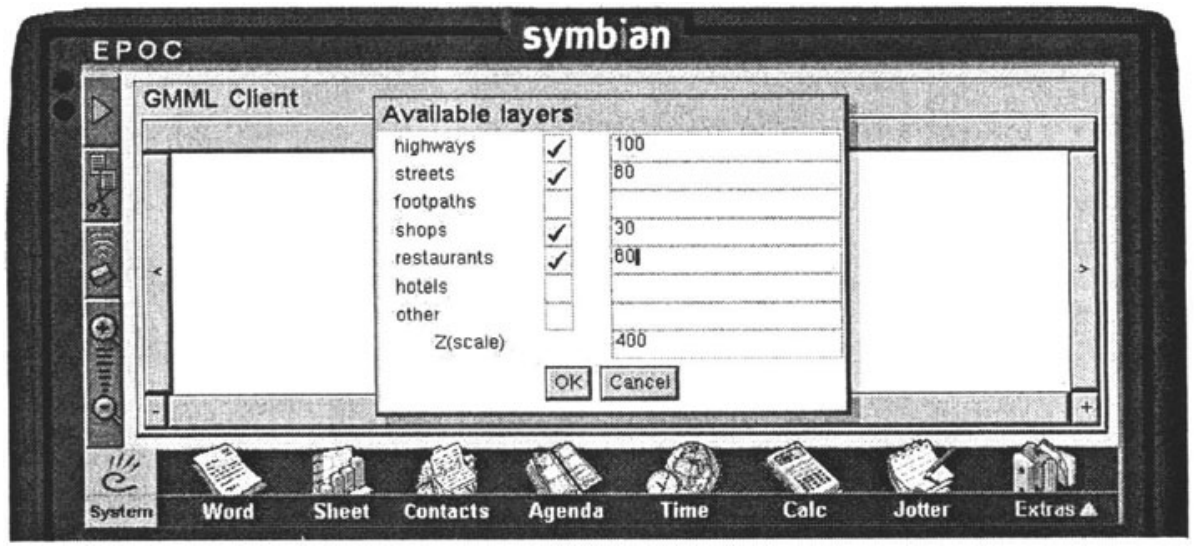

Figure 3. List of available data layers

After the user has selected the needed layers, defined the relative importance of them, and the preferred level of detail, the request for the data is made to the server. After retrieving data from the MLS server, it is presented to the user in the form of a map (figure 4). The current location of the user is also represented on the map and continuously updated. The interface supports basic operations like panning (long buttons on the borders of the screen) and zooming (small buttons in the down left and down right corners). The presentation of the map can be customised. The user can select colours and symbols to be used for representation. Some data layers can be excluded from the map. 


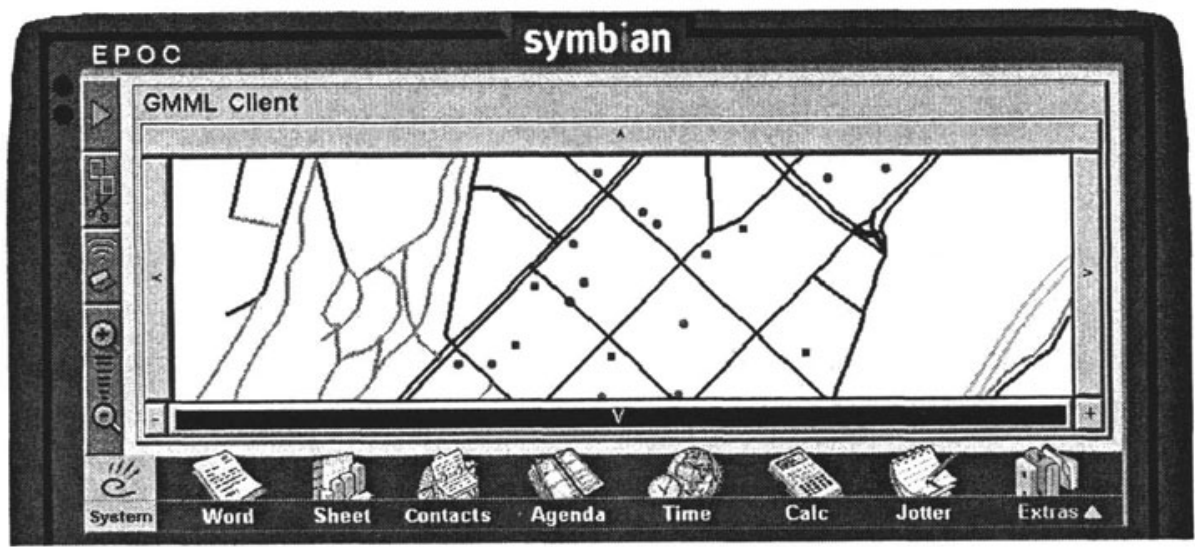

Figure 4. Map view

The user can retrieve information about a specific point of interest by pointing on the map (figure 5). As can be seen from the figure 5, the MLS client uses a separate view for point of interest presentation. Reason for this is, that the screen of a mobile terminal is usually too small to divide it into frames. User can also execute query for the nearest point of interest of a specified type (on the current stage, this query uses simple Euclid metric for selection of the nearest object).

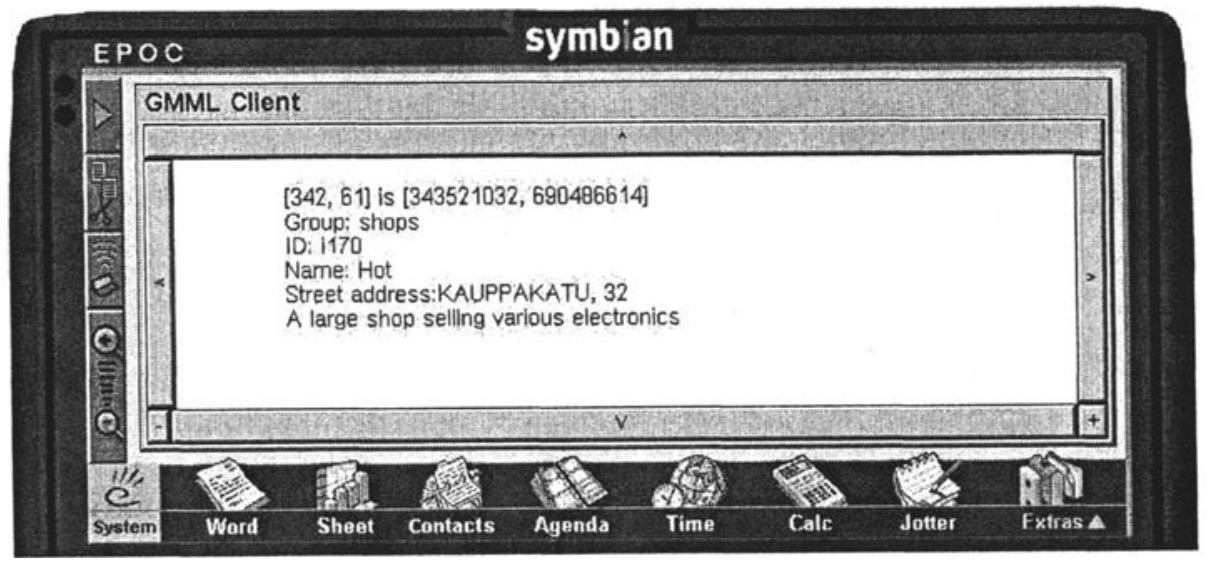

Figure 5. Point of interest description

Transaction Monitor component of the MLS client protects it from the unexpected disconnections and data losses. If a crash occurs, in most of cases the user is able to continue after restarting the application from the point where he was before the crash. When restoring a work session, the following routine is used. If the map data has already been received during 
this session, then the map is presented to the user. If only information about data layers is already available, then the application requests the present location of the user from the LCS and reconnects to the MLS server. Then the list of the layers (figure 3 ) is displayed then to the user. If no substantial data has been received (layers data or map data), then the session is closed.

\section{FUTURE WORK}

The MLS pilot system is still under development. The implemented basic features are to be complemented with more advanced features and methods. Some new solutions will also be implemented and tested. This is related to both, the client application and the architecture of the system in general.

In relation to the MLS client application, the future work is directed to the following tasks:

Improving the functionality and increasing the usability of the user interface.

Implementing analysis functions. Currently, the only function working is search for the nearest point of interest of a specified type, based on simple Euclid distance. A more advanced search algorithm, taking into account the road network, could be implemented. The search could also be made based on other parameters specified by the user (search by name, by value of an attribute etc.). A route finding algorithm should also be added.

Development of a version of the MLS client for J2ME. It could be interesting to test the application on a smartphone. When developing the client application, it was designed in a way that allows portability to other platforms, attempted to make it also as lightweight as possible. However, changes are needed at least to the user interface in order to port it to Java 2 Micro Edition.

- Further development of Transaction Monitor. On the current stage, TM is responsible primarily for data integrity on the client side. However, more research has to be done on questions related to the need to rollback an uncompleted transaction conducted via a mobile network and other related problems.

․ Moving to the standard Geography Markup Language (GML).

Development of GML is still going on. Therefore, this direction of future work strongly depends on the standardisation work held in the OpenGIS Consortium.

In relation to the server application of the MLS system, the main direction of the future work is further development of the data selection 
algorithm and implementation of progressive data transmission. The selection algorithm supports progressive transmission. However, there are open questions about removing data from a mobile terminal memory when the level of detail of a geographic feature is reduced (e.g. user moves out of the feature). A discussion on this topic can be found in Katasonov (2001).

In relation to the system component responsible for content, we could define the following directions of future work:

Integration of MLS with real location services.

- An effective exploitation of Oracle Spatial and probably other GIS software. Various spatial queries and analysis functions implemented in the GIS software could be utilised in the MLS system.

Providing dynamic information. An example of such information is information on the current traffic situation and roads condition.

- Providing information on mobile objects. Currently, the user is the only mobile object in the MLS system. In the present area, there are typically also other dynamic objects of interest, e.g. taxes, busses and other mobile users.

XML-based interaction with content servers. For flexibility and scalability reasons, the MLS server and content servers should be separated. Some XML-based data formats should be used in interaction between them. However, this direction of work depends on the standardisation process held in World Wide Web Consortium.

Another important direction of future work is testing the solutions of MLS in new environments. New mobile terminals with different operating systems are emerging and new mobile networks are appearing.

\section{ACKNOWLEDGEMENTS}

The present work was done in MultiMeetMobile research project [http://www.cs.jyu.fi/ mmm] at the Information Technology Research Institute, University of Jyväskylä, Finland. MultiMeetMobile project was financially supported by the National Technology Agency (TEKES) under contracts 40599/99, 40779/99, 40640/00, and industrial partners HewlettPackard, Nokia Networks and Yomi Vision.

\section{REFERENCES}

[1] Benefon, web site, http://www.benefon.com. 
[2] Burrough, P.A. and McDonnell, R.A., Principles of Geographical Information Systems, Oxford University Press, 1998.

[3] Clarke, K.C. "Cartography in a Mobile Internet Age", Proceedings of the 20th International Cartographic Conference, Beijing, China, August 2001, pp. 14811488.

[4] Garmash, A., Management of geographic information in mobile environment, M.Sc. thesis, University of Jyväskylä, Department of Computer Science and Information Systems, Jyväskylä, Finland, 2000.

[5] Gartner, G., Uhlirz, S. and Pammer, A. "Mobile Internet: Applying Maps to Mobile Clients", Proceedings of the Workshop on Maps and the Internet in the 20th International Cartographic Conference, Beijing, China, August 2001.

[6] Hardy, P.G., Haire, K.R., Sheehan, R. and Woodsford, P.A. "Mobile Mapping On-Demand, Using Active Representation and Generalisation", Proceedings of the 20th International Cartographic Conference, Beijing, China, August 2001, pp. 3239-3247.

[7] OpenGIS. Geography Markup Language (GML) 2.0, OpenGIS implementation specification, OGC document number 01-029, 20 February 2001. Available in http://www.opengis.net/gml/01-029/GML2.html.

[8] Katasonov, A., Intelligent user-centred transmission of geographical data in mobile location-based services, M.Sc. thesis, University of Jyväskylä, Department of Mathematical Information Technology, Jyväskylä, Finland, 2001.

[9] Krasner, G.E. and Pope, S.T., A cookbook for using the model-view-controller user interface paradigm in Smalltalk-80, Journal of Object-Oriented Programming, 1(3): pp. 26-49, 1988.

[10] kXML, web site http://kxml.enhydra.org

[11]Lehto, L. "XML in Web-based Geospatial Applications", Proceedings of the 3rd AGILE Conference on Geographic Information Science, Helsinki, Finland, May 2000.

[12]Lehto, L. and Kilpelainen, T. "Generalizing XML-Encoded Spatial Data on the Web", Proceedings of the 20th International Cartographic Conference, Beijing, China, August 2001.

[13] LIF, Location Interoperability forum, web site http://www.locationforum.org/

[14]Liikenne ja viestintäministeriö, Tie- ja katuverkon tietojärjestelmä DIGIROAD. Toteutussuunnitelma. 2001 , available in http://www.mintc.fi/www/sivut/dokumentit/julkaisu/mietinnot/2001/b16.html.

[15] Mims, P., Pilkington, P. and Blizzard, J. "An object oriented transfer format for the future", Proceedings of the ASPRS 2001 conference, St. Louis, USA, April 2001.

[16]Mitchell, K., "Wireless Mapping and Guidance Services", Proceedings of the 20th International Cartographic Conference, Beijing, China, August 2001, pp. $1785-1789$. 
[17]Reichenbacher, T. "The World in Your Pocket - Towards a Mobile Cartography", Proceedings of the 20th International Cartographic Conference, Beijing, China, August 2001, pp. 2514-2521.

[18] Singhal, S., Bridgman, T. Suryanarayana, L, Mauney, D., Alvinen, J., Bevis, D., Chan, J., and Hild, S., The Wireless Application Protocol, Addison-Wesley, Boston, 2001.

[19] Virrantaus, K., Veijalainen, J., Markkula, J., Katasonov, A., Garmash, A., Tirri, H., and Terziyan, V., Developing GIS-supported location-based services, Proceedings of the First International Workshop on Web Geographical Information Systems (WGIS), Kyoto, Japan, December 3-6, 2001, IEEE CS, 2001. 